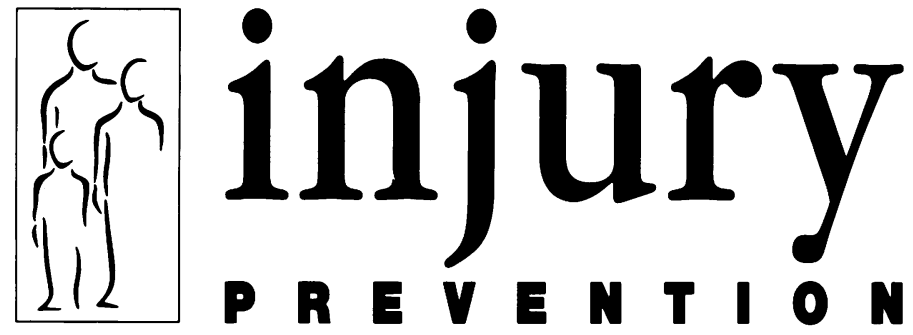

Journal of the International Society for Child and Adolescent Injury Prevention

\title{
Editorials
}

\section{The editor's job}

This seems a good opportunity to explain some aspects of the editor's job that may not be apparent to many readers or would-be-authors. My goal is to help others understand what lies behind most papers that appear in these pages. It is not a simple process by any stretch of the imagination.

When a paper arrives it is promptly logged into a special computerized tracking program (RMTS) and is given a manuscript number. Usually, within 24 hours an acknowledgment is sent to the corresponding author, stating that the paper will be reviewed in three to six weeks. Soon after, reviewers are assigned. Usually, one is chosen from the editorial board and a second (or occasionally, a third) is selected from the list of experts we have compiled. (There are now over 500 referees in our databank and many are previous authors.) Sometimes, a referee is chosen from among those listed in the references.

Referees are asked to make constructive comments and to recommend whether we should accept or reject papers they receive. They can suggest outright acceptance, accept pending revision, reject with a request for revision, or simply, reject. Most do what they are asked and do so in the time limit specified - three weeks for those in North America and a week or two longer for those from abroad. As a rule, the replies are thoughtful and helpful, both to me and the authors.

I then review each paper myself, and based on the referees' advice, return it to the author asking that the comments be addressed and the paper revised, or, with (what I hope is) a kind note, explaining why we do not intend to publish it. Usually I try to keep the door part way open for possible re-entry. Although as editor, I am heavily influenced by the reviewers, occasionally I see merit where they may not, and - much more rarely - the reverse is true.

Papers that are returned for revision are usually covered with scribbles, that, when deciphered, reflect my stylistic preferences. The most important of these is to ensure clarity, and fortunately, it is often the case that this is best achieved by cuts. This is just as well, because every editor is concerned with creating enough space to permit as many worthy papers to be published as promptly as possible.

Only one paper in the history of the journal has made it through this review process with no recommendations for revision by either reviewer, that is, a clear, unequivocal 'accept'. The exception is the paper by Sacks et al in this issue (p258). Even in this unusual instance, I could not resist making a few editorial suggestions that I hoped would improve the presentation, most of which the authors graciously accepted.

But most papers are returned with often detailed questions, comments, and suggestions. Unlike some of the purported advertising on public transit during the Olympics in Atlanta, Injury Prevention is just as proud of persistence as it is of 'winning'. So, for example, in this issue, there are three papers that underwent two rounds of revision and another that took three tries before the reviewers and I were satisfied. This amount of revision may be more than some authors will tolerate. One, reasonable reaction, is to say, 'To heck with it - let's try another journal'. Although I consider the stick-to-it decision more meritorious, I will not list the names of the dogged authors in case they view their behaviour in a different light. Nevertheless, I interpret their willingness to revise repeatedly as a great compliment to the journal; there are many other journals that present far fewer hassles for their contributors.

It is not embarrassing to admit that, as a new journal, we work harder with our authors to try to rescue papers that might otherwise be unacceptable. Only our readers can tell if we have succeeded. So, once again, we invite your comments. Criticisms are probably more helpful than compliments, but both are welcome.

Incidentally, as a quarterly, our publishing schedule is such that our absolute deadline is the date when the preceding issue appears in your mailbox. In other words, for the next issue (March 1997), all copy must be in the technical editor's hands by December 1996. This means that for an original article, that is, one that must be refereed (and revised) the latest reasonable date we could receive a submission and hope to have it published in the next issue is two months before this deadline. A more realistic average lag time is three or four months. This is a result of slow reviewers, a selfish editor (who will not give up an opera to edit a paper that arrived earlier that day), or authors who don't drop everything to revise when the markedup manuscript is returned. With all that we are proud to be able to offer a faster turnaround than most journals. 